\title{
Individual Behavioral Characteristics of Wild-Type Rats Predict Susceptibility to Experimental Autoimmune Encephalomyelitis
}

\author{
Annemieke Kavelaars*,1 Cobi J. Heijnen,* Ruth Tennekes,*, ${ }^{*}$ \\ Jan E. Bruggink, $\dagger$ and Jaap M. Koolhaas $\dagger$ \\ *Department of Immunology, University Children Hospital "Het Wilhelmina Kinderziekenhuis," \\ 3051 CA Utrecht; and †Department of Animal Physiology, University of Groningen, \\ Haren, The Netherlands
}

\begin{abstract}
Neuroendocrine-immune interactions are thought to be important in determining susceptibility to autoimmune disease. Animal studies have revealed that differences in susceptibility to experimental autoimmune encephalomyelitis (EAE) are related to reactivity in the hypothalamo-pituitary-adrenal axis. It is known that there is a close relation between neuroendocrine parameters and behavioral characteristics, suggesting that behavior and disease susceptibility may be associated. In the present study we investigated whether behavioral characteristics of wild-type rats are related to susceptibility to disease. We show here that the latency of the animal to attack an intruder correlates significantly with the EAE disease score: animals that do not attack the intruder during the test period are more resistant to the disease than animals with short attack latency times. These data, obtained in an unselected strain of wild-type rats, demonstrate that behavioral response patterns of individual animals can in part predict susceptibility to autoimmune disease. (๑) 1999 Academic Press
\end{abstract}

Key Words: experimental autoimmune encephalomyelitis; psychoneuroimmunology; wild-type rat; behavior; autoimmunity; aggressive behavior; HPA axis.

\section{INTRODUCTION}

Over the past years, it has become evident from animal research that genetic differences in neuroendocrine responses are associated with differences in susceptibility to autoimmune diseases. The first data in this area are from Sternberg et al., who investigated the differences in susceptibility to streptococcal cell wall-induced arthritis (SCW) between Lewis and Fischer rats (Sternberg, Hill, Chrousos, Kamilaris, Listwak, Gold, \& Wilder, 1989a). Lewis rats are highly susceptible to SCW arthritis and other autoimmune models, whereas Fischer rats are resistant. Investigation of the neuroendocrine reactivity in these two strains of rats demonstrated that the reactivity of the hypothalamo-pituitary-adrenal axis in Lewis rats is lower than in Fischer rats (Sternberg, Young, Bernardini, Calogero, Chrousos, Gold, \& Wilder, 1989b; Sternberg et al., 1989a). These differences in reactivity were observed after activation of the HPA axis by immune stimuli, e.g., injection with SCW or LPS, as well as after exposure to a novel environment (Sternberg et al., 1989a).

Evidence for a causal relation between HPA axis activity and susceptibility to disease was provided by demonstrating that treatment of Fischer rats with a glucocorticoid antagonist transforms them into SCW arthritis susceptible animals, whereas

${ }^{1}$ Address correspondence and reprint requests to Dr. Annemieke Kavelaars, Fax: 31-30-2320-712. e-mail: a.kavelaars@wkz.azu.nl. 
supplementation of Lewis rats with a glucocorticoid renders these animals resistant to the disease (Sternberg et al., 1989a).

More recently, we have demonstrated that the association between neuroendocrine response pattern and susceptibility to autoimmunity is not restricted to Lewis and Fischer rats. We examined susceptibility to experimental autoimmune encephalomyelitis (EAE) in two lines of Wistar rats (APO-sus and APO-unsus) that were selected on a pharmacogenetic basis with sensitivity to the dopaminergic agonist apomorphine as the selection criterium (Cools, Rots, Ellenbroek, \& De Kloet, 1993; Cools, Brachten, Heeren, Willemen, \& Ellenbroek, 1990). Our data demonstrated that APO-sus rats are resistant to EAE, whereas APO-unsus do get the disease. Similar differences in susceptibility to arthritis have been observed in these rats as well (Van de Langerijt, Van Lent, Hermus, Sweep, Cools, \& Van den Berg, 1994).

Further analysis of the immune response in APO-unsus and APO-sus rats revealed that the difference in susceptibility to autoimmunity is related to a difference on the level of $\mathrm{T}$ helper-1/T helper-2 (Th1/Th2) cytokine balance between rats from the two strains (Kavelaars, Heijnen, Ellenbroek, van Loveren, \& Cools, 1997a). In APOunsus rats that are sensitive to Th1-dependent autoimmune diseases like arthritis and EAE, the ratio of Th1 over Th2 cytokines produced after ex vivo stimulation is higher than in APO-sus rats (Kavelaars et al., 1997a). The two lines of rats show a dichotomy in the reactivity of the neuroendocrine system on the level of the HPA axis. APOsus rats have higher plasma ACTH levels at rest and in response to a stressor. In addition, corticosterone responses are higher and last longer in APO-sus rats than in APO-unsus rats (Rots, Cools, Oitzl, de Jong, Sutanto, \& De Kloet, 1996; Rots, Cools, de Jong, \& De Kloet, 1995).

It is well known in a wide variety of genetic selection lines that the differences in neuroendocrine response patterns are associated with differences in behavioral characteristics. For example, APO-sus Wistar rats that do not get EAE display higher locomotor responses in a novel environment than APO-unsus rats (Cools et al., 1993; Cools et al., 1990; Kavelaars et al., 1997). However, many strains and selection lines of laboratory rats are the product of rather intense breeding procedures and genetic selection processes. Therefore, one may wonder whether the observed relation among neuroendocrine response patterns, behavioral characteristics, and susceptibility to autoimmune disease is a more general phenomenon which can be observed in a normal population as well. To answer this question we used a strain of laboratory bred feral (wild-type) rats known to differ widely in their behavioral reactivity under a number of (social) environmental conditions. The attack latency in a resident-intruder paradigm was used as a reliable indicator of this individual differentiation in behavioral reactivity or coping style (Benus et al., 1998). The central question in the present study was whether behavioral characteristics may predict their susceptibility to autoimmunity. Animals were behaviorally characterized prior to inoculation with myelin basic protein emulsified in Freund's complete adjuvant for induction of EAE.

\section{METHODS}

Animals

Conventional male wild-type rats (Rattus norvegicus) bred in the Department of Animal Physiology, Groningen, The Netherlands, were used in this study. Animals were housed in groups of five individuals from weaning until the start of the experi- 
ments ( 4 months of age) in clear macrolon cages $(60 \times 40 \times 20 \mathrm{~cm})$ at ambient temperature on a 12-h light/12-h dark cycle. Bedding consisted of wood shavings, and food and water were available ad libitum. All experiments were performed in accordance to international and institutional guidelines for animal care.

\section{Determination of Attack Latency Time (ALT)}

Wild-type males were housed together with a sterilized wild-type female in wooden cages $(85 \times 60 \times 50 \mathrm{~cm})$ for 2 weeks. After these 2 weeks (after temporary removal of the female), each male animal was exposed to an intruder (male Wistar rat of $250 \pm 25 \mathrm{~g}$ ) in a standard resident-intruder test (Koolhaas, Schuurman, \& Wiepkema, 1980) on 4 consecutive days. The latency to first attack (in seconds) was determined and the intruder was removed immediately. When animals did not attack the intruder within $600 \mathrm{~s}$, the test was ended by removal of the intruder. The ALT represents the average of the four daily trials.

\section{Experimental Autoimmune Encephalomyelitis}

Experimental autoimmune encephalitis was induced in a total of 31 animals in two separate experiments. Data reflect combined results of these two experiments. One animal died after inoculation with myelin basic protein (MBP) in FCA.

Two weeks after the determination of the ALT, animals were inoculated in the hind paw with $100 \mu \mathrm{l}$ of inoculate under brief halothane anesthesia. The inoculate consisted of $2 \mathrm{mg}$ myelin basic protein isolated from guinea pig brain in $1 \mathrm{ml}$ saline mixed with $1 \mathrm{ml}$ complete Freund's adjuvant (Difco, Detroit, MI) to which $10 \mathrm{mg}$ mycobacterium tuberculosis $\mathrm{H} 37 \mathrm{Ra}$ was added. Rats were examined daily to score the development of clinical signs of the disease. Clinical signs were scored on a scale from 0 to 7: 0 , no disease; 1 , loss of tail tonicity; 2 , paralysis tail; 3 , hind paw weakness; 4, severe locomotor problems; 5, paralysis of both hind limbs; 6, paralysis up to diaphragm; 7 , death as a result of EAE.

\section{Determination of HPA Axis reactivity}

One week after the determination of the ALT, animals were provided with a silastic heart cannula (id $0.5 \mathrm{~mm}$; od $0.9 \mathrm{~mm}$; Dow Corning) through the right jugular vein under Hypnorm anesthesia (10 mg/kg fluanisone and $0.2 \mathrm{mg} / \mathrm{kg}$ fentanyl ip; Janssen Pharamceutica) after premedication with atropine $(1 \mathrm{mg} / \mathrm{kg}$, ip) and diazepam $(5 \mathrm{mg} /$ $\mathrm{kg}$ ip). The canula was externalized on the top of the skull as described (Steffens, 1969) to allow frequent blood sampling in conscious, undisturbed freely moving rats (Wiersma \& Kastelijn, 1985; Steffens, 1969).

After surgery, animals were allowed to recover for 10 days and habituated to the sampling procedure. Blood samples were taken at baseline $(t=-10 \mathrm{~min})$ and at 30 , 60,90 , and $120 \mathrm{~min}$ after administration of CRH. CRH (500 ng/kg body wt) was administered iv via the canula. This dose of $\mathrm{CRH}$ has been used successfully to detect individual differences in HPA axis reactivity (Ruis, Ter Brake, Buwalda, De Boer, Meerlo, Korte, Blokhuis, \& Koolhaas, 1998; Buwalda, De Boer, Donne Schmidt, Felszeghi, Nyakas, Sgoifo, van der Vegt, Tilders, Bohus, \& Koolhaas, 1998).

Plasma corticosterone was determined by reversed-phase high-performance liquid chromatography (Dawson, Kontur, \& Monjan, 1984). 


\section{Data Analysis}

Data were analyzed by Spearman rank correlation test or ANOVA, and $p<.05$ was considered statistically significant.

\section{RESULTS}

\section{Behavioral Characteristics of Wild-Type Rats Used for Induction of EAE}

Animals were characterized behaviorally by determining the latency to attack an intruder in a standard resident-intruder test. In our test group, attack latency times determined as the average of four trials on consecutive days varied between 82 and $600 \mathrm{~s}$. Nine of 31 animals did not attack the intruder in any of the four trials. For the other 22 animals median attack latency time was 362 s (range 82-567 s).

\section{Relation between Disease Score and ALT}

To determine whether there is a relation between ALT and susceptibility to autoimmunity, all animals were inoculated with MBP in FCA 14 days after determination of ALT. Clinical symptoms of the disease were scored daily. First symptoms were observed on days 9 or 10 after inoculation. Maximal disease activity occurred on day 14, after which the animals gradually recovered. Total disease scores were determined by summation of the daily scores for each individual animal. The data in Fig. 1 demonstrate that there is a significant inverse relation between ALT and total disease score (two-tailed Spearman rank correlation test: $r=-0.54, n=30, p=.002$ ).

To determine whether the difference in total disease score is due to differences in the course of the disease, we also analyzed mean disease scores in two groups of animals: passive animals defined as animals with the maximal ALT of $600(n=$ $9)$ and active animals with ALT in the lower 30th percentile $(n=10)$. As depicted in Fig. 2, there were no differences with respect to day of onset, peak or duration of the disease. However, disease severity did differ significantly between these two groups

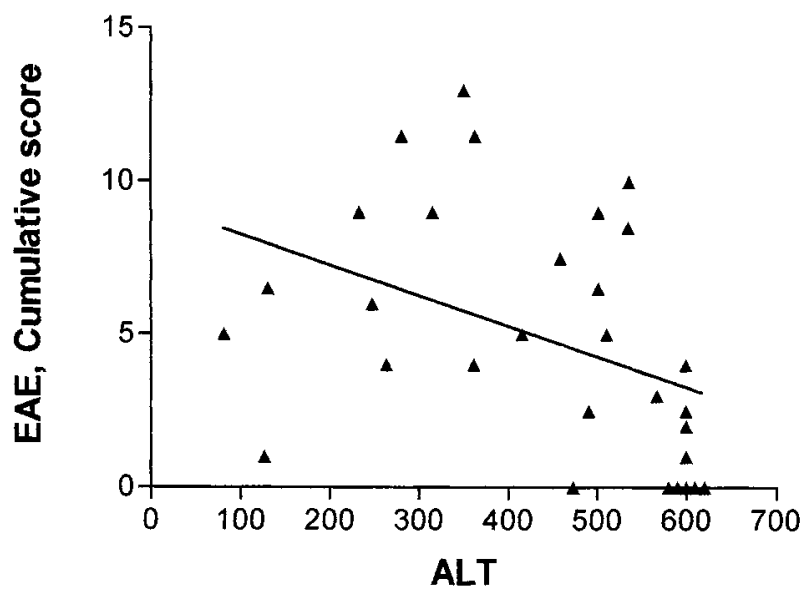

FIG. 1. Relation between ALT and disease score. Attack latency times were determined using a standard resident-intruder test (see Methods). Two weeks after the last resident-intruder test, animals were inoculated with MBP in FCA in the hind paw. Clinical signs of disease were scored daily and total disease scores for each animal were determined by summation of the score of each day. Spearman rank correlation test for ALT $\times$ disease score: $r=-.54, n=30$, two-tailed $p=.002$. 


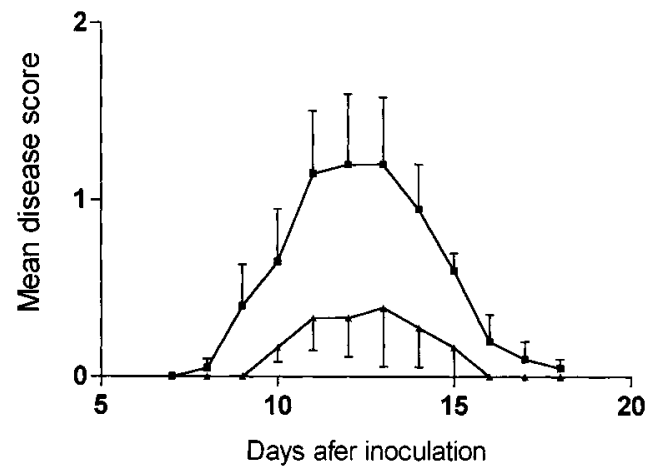

FIG. 2. Course of EAE. Attack latency times were determined using a standard resident-intruder test and disease was induced as described in the legend to Fig. 1. Data represent mean disease scores for animals with maximal ALT (ALT $600 \mathrm{~s}, n=9)(\boldsymbol{\Delta})$ and active animals with ALT in the lower 30th percentile $(n=10, \mathbf{\square})$. ANOVA $(F=21.4 ; p<.001$.

(ANOVA: group effect, $F(1,198)=21.4 ; p<.001 ;$ time effect: $F(11,198)=4.38$, $p<.001$; Interaction effect: $F(11,198)=1.15 ; p=.33)$.

\section{Reactivity of the HPA Axis}

In a second (new) group of animals, to determine whether ALT are related to the reactivity of the HPA axis we measured the corticosterone response to administration of CRH. Our data demonstrate that there was no correlation between ALT and corticosterone response determined as area under the curve (not shown). Moreover, there was no difference in the time course of the corticosterone response between animals with the maximal ALT and animals in the lower 30th percentile (Fig. 3).

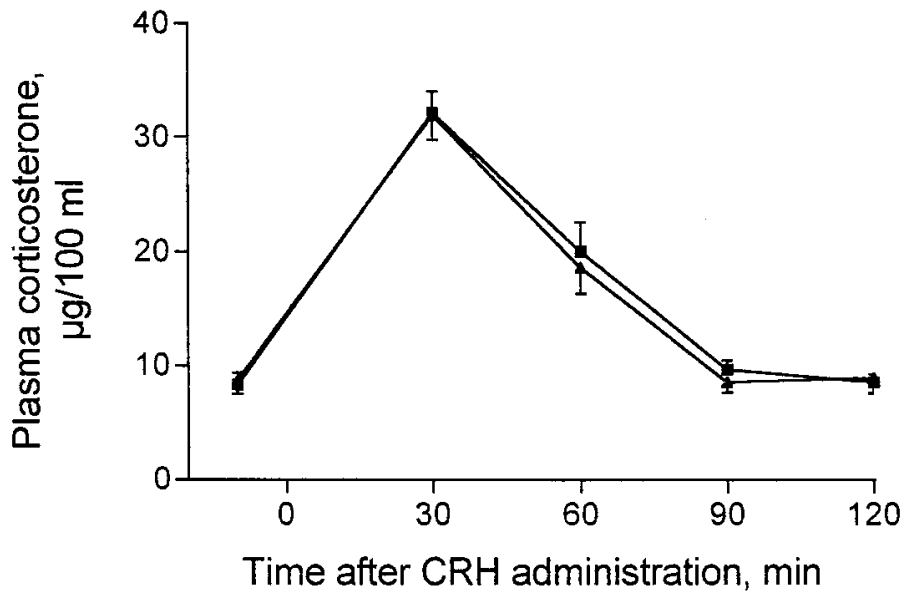

FIG. 3. Corticosterone response to CRH. Attack latency times (ALT) were determined using a standard resident-intruder test. One week later, animals were provided with a heart cannula that was externalized on the skull to allow blood sampling. After 10 days recovery, blood samples were taken at the indicated time periods after iv administration of CRH $(500 \mathrm{ng} / \mathrm{kg}$ body wt). Plasma corticosterone levels were determined. Data represent plasma corticosterone levels for animals with maximal ALT (ALT 600 $\mathrm{s}, n=12)(\mathbf{\Delta})$ and active animals with ALT in the lower 30th percentile $(n=14, \boldsymbol{\square})$. ANOVA: $F(1,120)$ $=.14, p=.71$. 


\section{DISCUSSION}

In an outbred strain of wild-type rats, we demonstrated that behavioral characteristics in a resident-intruder paradigm partially predict the severity of EAE in these rats. Our data show that aggressive rats (short ALT) are more susceptible to EAE than nonaggressive rats (ALT $600 \mathrm{~s}$ ), who hardly get the disease. The ALT in a resident-intruder test is indicative for the level of aggressive behavior and coping style of the individual rat (Koolhaas \& Bohus, 1995). Hence, in an unselected population of wild-type rats, the large individual susceptibility to autoimmune disease correlates with the individual capacity to cope with environmental challenges.

From previous research, we know that there is a strong correlation between the level of aggressive behavior in these wild-type rats and the neuroendocrine response to a number of stressors. Exposure of the animals to a defeat stress or to a shock prod results in sympathetic, adrenomedullary, and adrenocortical reactivity (Sgoifo, De Boer, Haller, \& Koolhaas, 1996). The reactivity of the sympathetic (plasma noradrenaline) and adrenomedullary (plasma adrenaline) system can be predicted on the basis of the ALT. In animals with short ALT, i.e., aggressive animals, stress-induced increases in plasma adrenaline and noradrenaline are much higher than in animals with ALT of $600 \mathrm{~s}$ (Sgoifo et al., 1996). However, the reactivity of the HPA axis determined as plasma corticosterone responses to either shock prod burying or defeat was not related to attack latency times. This finding is in line with our present data showing that there is no relation between ALT and corticosterone response after administration of $\mathrm{CRH}$. We also have preliminary data suggesting that LPS-induced corticosterone responses are not related to ALT. Therefore, we conclude that the relation between ALT and EAE susceptibility is not simply a reflection of differences in HPA axis activity. These data are in contrast to what has been observed in Fischer versus Lewis rats or when comparing APO-sus and APO-unsus Wistar rats. In these strains or lines of rats, susceptibility to autoimmune disease coincides with blunted reactivity of the HPA axis.

If reactivity of the HPA axis is not related to disease susceptibility in the more aggressive rats, which other systems are involved? One possibility would be that the increased sympatho-adrenomedullary reactivity of the susceptible animals plays a role. Catecholamines can modulate the activity of the immune system in several ways. Lymphoid cells express $\alpha$ - and $\beta$-adrenergic receptors, triggering of which can result in alterations of cytokine production and thereby may contribute to disease activity (Khan et al., 1986; Bisphoric, Cohen, \& Lefkowitz, 1980; Van der Poll, Coyle, Barbosa, Braxton, \& Lowry, 1996; Heijnen, Rouppe van der Voort, Wulffraat, van der Net, Kuis, \& Kavelaars, 1996; Spengler, Allen, Remick, Strieter, \& Kunkel, 1990; Spengler, Chensue, Giacherio, Blenk, \& Kunkel, 1994). In general, $\beta 2$-adrenergic receptor agonists will inhibit proinflammatory cytokine production (Van der Poll et al., 1996). However, recently we demonstrated that the production of the proinflammatory cytokine IL-8 is enhanced by addition of a $\beta 2$-adrenergic agonist (Kavelaars, Van de Pol, Zijlstra, \& Heijnen, 1997). Moreover, it has been shown that administration of the $\alpha 1$-adrenergic receptor antagonist prazosin results in decreased EAE symptoms (Brosnan, Goldmutz, Cammer, Factor, Bloom, \& Norton, 1985). Thus, it is conceivable that higher levels of noradrenaline enhance disease symptoms, probably via $\alpha$-adrenergic receptors giving rise to upregulation of proinflammatory cytokines (Khan et al., 1986; Bisphoric, Cohen, \& Lefkowitz, 1980; Van der Poll et al., 
1996). This interpretation may be supported by recent evidence that the differential sympathetic reactivity in the wild-type rat is partially due to a less functional presynaptic and postsynaptic $\beta 2$-adrenergic receptor in the nonaggressive male (Smit, van Oosten, Palm, Doze, Koolhaas, \& Zaagsma, 1998).

In summary, we have demonstrated that behavioral characteristics in wild-type rats correlate with susceptibility to EAE. In these animals, there is no evidence for a relation between reactivity of the HPA axis and disease. Further research would be needed to determine to what extent the differential EAE susceptibility might be explained by the differential reactivity of the sympatho-adrenomedullary system. The possibility should be considered, however, that more than one neuroendocrine pathway will contribute to the susceptibility to autoimmune disease.

\section{ACKNOWLEDGMENTS}

This study was supported in part (R.T. and J.B.) by NWO grant 904-64-021.

\section{REFERENCES}

Benus, R. F., Bohus, B., Koolhaas, J. M., \& Oortmersen, G. A. v. (1998). Heritable variation in aggression as a reflection of individual coping strategies. Experientia 47, 1008-1019.

Bisphoric, N. H., Cohen, H. J., \& Lefkowitz, R. J. (1980). Beta-adrenergic receptors in lymphocyte subpopulations. J. Allergy Clin. Immunol., 65, 29-33.

Brosnan, C. F., Goldmutz, E. A., Cammer, W., Factor, S. M., Bloom, B. R., \& Norton, W. T. (1985). Prazosin, an $\alpha 1$-adrenergic receptor antagonist, suppresses experimental autoimmune encephalomyelitis in the Lewis rat. Proc. Natl. Acad. Sci. USA 82, 5915-5919.

Buwalda, B., De Boer, S. F., Donne Schmidt, E., Felszeghi, K., Nyakas, C., Sgoifo, A., van der Vegt, B. J., Tilders, F. J. H., Bohus, B., \& Koolhaas, J. M. (1999). Long lasting deficient dexamethasone suppression of HPA activation following peripheral CRF challenge in socially defeated rats. $\mathrm{J} \mathrm{Neu-}$ roendocrinol., in press.

Cools, A. R., Brachten, R., Heeren, D., Willemen, A., \& Ellenbroek, B. (1990). Search after neurobiological profile of individual-specific features of Wistar rats. Brain Res. Bull. 24, 49-69.

Cools, A. R., Rots, N. Y., Ellenbroek, B., \& De Kloet, E. R. (1993). Bimodal shape of individual variation in behaviour of Wistar rats: the overall outcome of a fundamentally different make-up and reactivity of the brain, the endocrinological and the immunological system. Neuropsychobiology 28, 100105.

Dawson, J. R., Kontur, P., \& Monjan, A. (1984). High-performance liquid chromatographic (HPLC) separation and quantitation of endogenous glucocorticoids after solid-phase extraction from plasma. Hormone Res. 20, 89-94.

Heijnen, C. J., Rouppe van der Voort, C., Wulffraat, N., van der Net, J., Kuis, W., \& Kavelaars, A. (1996). Functional $\alpha 1$-adrenergic receptors on leukocytes of patients with polyarticular juvenile rheumatoid arthritis. J. Neuroimmunol. 71, 223-226.

Kavelaars, A., Heijnen, C. J., Ellenbroek, B., van Loveren, H., \& Cools, A. R. (1997). Apomorphinesusceptible and apo-morphine-unsusceptible Wistar rats differ in their susceptibility for inflammatory and infectious diseases: A study on rats with groupific differences in structure reactivity of hypothalamic-pituitary-adrenal axis. J. Neurosci. 17, 2580-2584.

Kavelaars, A., Van de Pol, M., Zijlstra, J., \& Heijnen, C. J. (1997). $\beta 2$-adrenergic receptor activation stimulates the production of IL-8 by human monocytes. J. Neuroimmunol. 77, 211-216.

Khan, M. M., Sansoni, P., Silverman, E. D., Engleman, E. G., \& Melmon, K. L. (1986). Beta-adrenergic receptors on human suppressor, helper, and cytolytic lymphocytes. Biochem. Pharmacol. 35, 11371142.

Koolhaas, J. M., \& Bohus, B. (1995). Animals models of stress of immunity. In B. Leonard \& K. Miller (Eds.), Stress, the immune system and psychiatry, pp. 69-83. Wiley: New York. 
Koolhaas, J. M., Schuurman, T., \& Wiepkema, P. R. (1980). The organization of intraspecific agonistic behavior in the rat. Prog. Neurobiol. 15, 247-268.

Rots, N. Y., Cools, A. R., de Jong, J., \& De Kloet, E. R. (1995). Corticosteroid feedback resistance in rats genetically selected for increased dopamine responsiveness. J. Neuroendocrinol. 7, 153-161.

Rots, N. Y., Cools, A. R., Oitzl, M. S., de Jong, J., Sutanto, W., \& De Kloet, E. R. (1996). Divergent prolactin and pituitary-adrenal activity in rats selectively bred for different dopamine responsiveness. Endocrinology 137, 1678-1686.

Ruis, M. A. W., Ter Brake, J. H. A., Buwalda, B., De Boer, S. F., Meerlo, P., Korte, S. M., Blokhuis, H. J., \& Koolhaas, J. M. (1999). Housing familiar male wildtype rats together reduces the longterm adverse behavioral and physiological effects of social defeat. Psychoneuroendocrinology, in press.

Sgoifo, A., De Boer, S. F., Haller, J., \& Koolhaas, J. M. (1996). Individual differences in plasma catecholamine and corticosterone stress responses of wild-type rats: relationship with aggression. Physiol. Behav. 60, 1403-1407.

Smit, J., van Oosten, R. V., Palm, I. F., Doze, P., Koolhaas, J. M., \& Zaagsma, J. (1998). Prejunctional modulation of noradrenergic neurotransmission in wild-type rat (Rattus norvegicus); A possible relationship with coping styles. Am. J. Physiol., in press.

Spengler, R. N., Allen, R. M., Remick, R. M., Strieter, R. M., \& Kunkel, S. L. (1990). Stimulation of $\alpha$-adrenergic receptor augments the production of macrophage-derived tumor necrosis factor. $J$. Immunol. 145, 1430-1434.

Spengler, R. N., Chensue, S. W., Giacherio, G. A., Blenk, N., \& Kunkel, S. L. (1994). Endogenous norepinephrine regulates tumor necrosis factor- $\alpha$ production from macrophages in vitro. Journal of Immunology 152, 3024-3031.

Steffens, A. B. (1969). A method for frequent sampling of blood and continuous infusion of fluids in the rat without disturbing the animal. Physiol. Behav. 4, 833-836.

Sternberg, E. M., Hill, J. M., Chrousos, G. P., Kamilaris, T., Listwak, S. J., Gold, P. W., \& Wilder, R. L. (1989a). Inflammatory mediator-induced hypothalamic-pituitary-adrenal axis activaion is defective in streptococcal cell wall arthritis-susceptible Lewis rat. Proc. Natl. Acad. Sci. USA 86, 2374-2378.

Sternberg, E. M., Young, W. S., Bernardini, R., Calogero, A. E., Chrousos, G. P., Gold, P. W., \& Wilder, R. L. (1989b). A central nervous system defect in biosynthesis of corticotropin releasing hormone is associated with susceptibility to streptococcal cell wall-induced arthritis in Lewis rats. Proc. Natl. Acad. Sci. USA 86, 4771-4775.

Van de Langerijt, A. G., Van Lent, P. L., Hermus, A. R., Sweep, C. G., Cools, A. R., \& Van den Berg, W. B. (1994). Susceptibility to adjuvant arthritis: Relative importance of adrenal activity and bacterial flora. Clin. Exp. Immunol. 97, 33-38.

Van der Poll, T., Coyle, S. M., Barbosa, K., Braxton, C. C., \& Lowry, S. F. (1996). Epinephrine inhibits tumor necrosis factor- $\alpha$ and potentiates interleukin-10 production during human endotoxemia. $J$. Clin. Invest. 97, 713-719.

Wiersma, J., \& Kastelijn, J. (1985). A chronic technique for high frequency blood sampling/transfusion in the freely behaving rat which does not affect prolactin and corticosterone secretion. J. Endocrinol. 107, 285-292.

Received August 17, 1998 